\title{
Post deposition nitridation of Si in W/Si soft X-ray multilayer systems
}

\author{
R.V. Medvedev ${ }^{\text {a, }}$, C.P. Hendrikx ${ }^{\text {a }}$, J.M. Sturm ${ }^{\text {a }}$, S.N. Yakunin ${ }^{\text {b }}$, I.A. Makhotkin ${ }^{\text {a }}$, A.E. Yakshin ${ }^{\text {, }}$, \\ F. Bijkerk ${ }^{\mathrm{a}}$
}

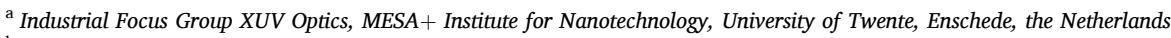

${ }^{\mathrm{b}}$ NRC Kurchatov Institute, Moscow, Russia

A R T I C L E I N F O

\section{Keywords:}

$2.5 \mathrm{~nm}$

$0.84 \mathrm{~nm}$

\begin{abstract}
A B S T R A C T
Partial nitridation of $\mathrm{W} / \mathrm{Si}$ multilayer systems with a $\mathrm{nm}$ period was investigated in an attempt to reduce optically unfavorable tungsten silicide formation in the systems. Nitridation was applied directly after the deposition of every Si layer by exposing the Si surfaces to reactive nitrogen species from an ion source. As a result the formation of substantially sharper interfaces and a reduction in the silicide formation were observed in the systems. However, the passivation treatment caused a pronounced reduction in the X-ray reflectivity at $0.84 \mathrm{~nm}$ wavelength because of deep nitrogen penetration in the Si layers. This reduced the optical contrast between the reflector and spacer layer, which compensated the effect of the improved interface profile.
\end{abstract}

\section{Introduction}

$\mathrm{W} / \mathrm{Si}$ short-period multilayer systems (of about $2.5 \mathrm{~nm}$ ) are widely used in X-ray fluorescence (XRF) applications to achieve a high reflectivity in the wavelength range $0.84 \mathrm{~nm}-2.4 \mathrm{~nm}$, which corresponds to spectral lines of $\mathrm{O} \mathrm{K} \alpha$ to $\mathrm{Al} \mathrm{K} \alpha$. The advantage of using multilayer mirrors is the ability to tune them to a specific wavelength required for a specific task. The shorter periods allow for a larger angular distance between the spectral peaks, which is beneficial for the analysis in XRF applications as it prevents overlapping of the peaks of interest. However, the shortperiod W/Si multilayer systems have limited reflectivity, which limits the sensitivity of XRF analysis. Currently the highest achieved reflectivity at $0.84 \mathrm{~nm}$ for $\mathrm{W} / \mathrm{Si}$ is $40 \%$, while the theoretical reflectivity is $60 \%$ for an ideal system that presumes no interfacial roughness or interdiffusion [1]. Roughness has significant effect on the optical contrast and therefore the reflectivity of these short-period multilayers. However, recent attempts to reduce it to below the currently achieved level of about $0.3 \mathrm{~nm}$ was not successful [1]. Interdiffusion is another factor that significantly reduces the reflectivity in short-period multilayers. It was found that a tungsten silicide compound is formed in W/Si multilayers that reduces the optical contrast considerably [1,2]. The preliminary research revealed that the $\mathrm{W}$-on-Si interface was proven to be significantly broader than the Si-on-W interface [3]. Therefore, research is needed on the improvement of the $\mathrm{W}$-on-Si interface in the first place. In this work we used passivation of the Si layer surface with nitrogen as one of the possible solutions to prevent chemical interaction between the two materials. Passivation is used in many applications to prevent unwanted compound formations. In microelectronics passivation is used to improve lifetime and quality of elements, while in thin-film transistors it is used to create barrier layers preventing chemical interactions [4-6]. In X-ray optics, the definition of the layer materials was efficiently improved, for example, by nitridation of every La layer, as in La/B multilayers [7-9]. Other examples of a successful nitridation was shown for $\mathrm{Ru} / \mathrm{B}_{4} \mathrm{C}, \mathrm{Pd} / \mathrm{B}_{4} \mathrm{C}$ and $\mathrm{Co} / \mathrm{C}$ multilayer systems [10-14]. A $\mathrm{Si}_{3} \mathrm{~N}_{4}$ compound could be used to prevent W-Si interaction, while nitridation of Si was reported before in [15-17]. The nitridation was implemented by reactive sputtering of $\mathrm{Si}$ or $\mathrm{Si}_{3} \mathrm{~N}_{4}$ in $\mathrm{N}_{2}$ or applying ion assisted deposition using an ion source supplied with $\mathrm{N}_{2}$. These studies were devoted to the passivation of the entire Si layer to obtain silicon nitride. However, for our application of W/Si at $0.84-2.4 \mathrm{~nm}$, passivation of the entire Si layer is optically unfavorable. To avoid reflectivity loss in our case the thickness of $\mathrm{Si}_{3} \mathrm{~N}_{4}$ at the W-on-Si interface should not exceed $0.3 \mathrm{~nm}$. For this reason, in this work an attempt was made to nitridate only the surface of the Si layers by post deposition low energy ion treatment and to avoid full nitridation of the Si layers.

\footnotetext{
* Corresponding author.

E-mail address: r.medvedev@utwente.nl (R.V. Medvedev).
} 


\section{Experimental}

\subsection{Multilayer deposition}

The multilayer mirrors were deposited on super-polished silicon wafers of $25 \mathrm{~mm} \times 25 \mathrm{~mm}$ (root mean square roughness of about 0.14 $\mathrm{nm}$ ) using DC magnetron sputtering in a system with a base pressure of $1 \times 10^{-7} \mathrm{~Pa}$. Ar gas was used as a working gas at a pressure of about $1 \times$ $10^{-1} \mathrm{~Pa}$ (Ar flow of $26 \mathrm{sccm}$ ). Depositions were performed at a power of $84 \mathrm{~W}$ for $\mathrm{W}$ and $213 \mathrm{~W}$ for Si. Tungsten was deposited with a low power in order to have a better control on thickness. Deposition rates were $0.075 \mathrm{~nm} / \mathrm{s}$ and $0.02 \mathrm{~nm} / \mathrm{s}$ for Si and W, respectively. An ion source was used for passivation of the Si layers. The ion source was fed with $\mathrm{Ar}$ (flow of $14 \mathrm{sccm}$ ) and $\mathrm{N}_{2}$ (flow of $7 \mathrm{sccm}$ ). The ion source was mounted at 60 degrees to the substrate at a distance of $70 \mathrm{~cm}$. The following parameters were used: ion beam voltage of $60 \mathrm{eV}$, and ion current of $120 \mathrm{~mA}$. The multilayer mirrors had a period of $2.5 \mathrm{~nm}$ and a gamma value $\left(\Gamma=d_{\mathrm{W}} /\right.$ $\left.d_{\mathrm{W}}+d_{\mathrm{Si}}\right) \Gamma=0.12$. The thickness of the Si capping layer was the same as in the entire multilayer stack $d_{\mathrm{Si}}=2.2 \mathrm{~nm}$. The main set of the experimental multilayers consisted of 50 periods as it allowed for analysis that is more accurate. A selected number of multilayers consisted of 200 periods in order to achieve the maximum reflectivity. To avoid oxidation of the surfaces after exposure to the air, every mirror was finished with a $\mathrm{Si}$ layer with the same thickness as inside the stack. Uniformity of the deposition and ion treatment was achieved by rotating the holder with substrates at $60 \mathrm{rpm}$ during the entire deposition process. Configuration of the set-up is described elsewhere [18].

\subsection{Multilayer characterization}

Soft X-ray reflectivity at the specific wavelength and grazing angle of incidence required for the application $\left(0.84 \mathrm{~nm}, \theta \approx 9.7^{\circ}\right.$ with respect to the surface) were measured at the storage ring BESSY II at the Physikalisch-Technische Bundesanstalt (PTB) in Berlin, Germany.

For a qualitative assessment of the roughness of the multilayer systems, X-ray diffused scattering rocking curves were measured with a conventional X-ray tube at the wavelength of $0.154 \mathrm{~nm}$. The rocking curves were taken at the second Bragg peak. The procedure of the measurement is described elsewhere [19].

The subsurface composition of the samples was determined by nondestructive X-ray Photoelectron Spectroscopy (XPS). A Thermo Scientific Theta Probe Instrument was used employing monochromatic Al-Ka radiation, providing a typical full-width half maximum of the $\mathrm{Ag} 3 \mathrm{~d}_{5 / 2}$ peak equal to $0.75 \mathrm{eV}$, as indication for the obtained energy resolution. The probing depth of the non-destructive measurement is about $6 \mathrm{~nm}$. The photoelectron signal is integrated over the entire acceptance angle of the analyzer, corresponding to $\pm 30^{\circ}$ around an average take-off angle of $53^{\circ}$ with respect to the surface normal. Spectra were evaluated with Thermo-Avantage software, applying quantification based on analyzer transmission, Scofield sensitivity factors and inelastic electron mean free path values calculated according to the method of Cumpson and Seah [20]. The non-destructive measurement of subsurface composition was done after exposure of the samples to the air. In-depth distribution of the elements was assessed by XPS sputter depth-profiling with $0.25 \mathrm{keV} \mathrm{Ar}^{+}$ ions.

Grazing incidence X-ray reflectivity (GIXR) and X-ray fluorescence from W modulated by an X-ray standing wave (F-XSW) in the multilayer were measured using an in-house Malvern Panalytical Empyrean X-ray diffractometer using a Cu- $\mathrm{K}_{\alpha}$ source (1.5406 ̊) [21]. A 4xGe 220 monochromator was used between the source and the sample. The measurements were performed in two steps. First, the X-ray reflectivity curves were measured up to 8 degrees incidence. These measurements were performed in continuous scanning mode using various steps sizes and accumulation times in order to compromise between good measurement statistics for higher order Bragg peaks and reasonable total measurement time (Table 1). At the second step X-ray fluorescence was
Table 1

XRR measurement configuration.

\begin{tabular}{lll}
\hline Range, deg. & Step size, deg. & Accumulation time per point, s. \\
\hline $0.1-1.32$ & 0.004 & 3.5 \\
$1.32-2.8$ & 0.005 & 4.4 \\
$2.8-8$ & 0.008 & 8.8 \\
\hline
\end{tabular}

measured as a function of the X-ray angle of incidence on the sample. To capture these signals, we have equipped the diffractometer with an Amptek energy dispersive XR-100SDD Silicon Drift Detector. Only the angular range corresponding to the first Bragg peak was scanned since for larger angles fluorescence modulation could not be detected in the used measurement setup. The measurements were performed with an angular step of 0.0025 degree around the Bragg peak and 0.005 degrees outside the Bragg peak. To accumulate a sufficient number of fluorescent photons, the accumulation time was 5 min per angular step.

The data analysis was performed using a free-form approach similar to the one discussed in [22]. Within this approach the periodically repeated W-Si bi-layers were divided into a set of $0.3 \mathrm{~nm}$ thick sublayers [21]. While the thickness of the sublayers is fixed, the optical constant of each individual sublayer is varied during the fitting of GIXR data. A minimizing procedure for cumulative mismatch between the measured and simulated data was applied for both GIXR and F-XSW simultaneously.

\section{Results}

\subsection{Passivation of $\mathrm{Si}$}

In our preliminary research the $\mathrm{W}$-on-Si interface was found to be significantly broader than the Si-on-W interface in $2.5 \mathrm{~nm}$ W/Si multilayers [3]. This was due to a $\mathrm{W}$ silicide formation during $\mathrm{W}$ deposition on $\mathrm{Si}$. To minimize the interaction at the W-on-Si interface, the top part of every $\mathrm{Si}$ layer was passivated with nitrogen. Ideally, a silicon nitride layer with a thickness of about $0.3 \mathrm{~nm}$ on top of the Si layer was to be formed, as illustrated in Fig. 1.

According to simulations a layer of $0.3 \mathrm{~nm}$ of $\mathrm{Si}_{3} \mathrm{~N}_{4}$ would only slightly reduce reflectivity ( $R=58.8 \%$ ) compared to the ideal bi-layer $\mathrm{W} / \mathrm{Si}$ system $(R=60 \%)$. A thicker layer of silicon nitride would already affect the reflectivity too strongly due a reduced optical contrast at $0.84 \mathrm{~nm}$. Note that a $\mathrm{W}$ silicide layer formed in $\mathrm{W} / \mathrm{Si}$ multilayers reduces reflectivity by about 10 abs. \%.To implement nitridation, the surface of every Si layer (after its deposition) was exposed to reactive nitrogen species from the ion source. The growth mechanism of $\mathrm{Si}_{3} \mathrm{~N}_{4}$ was not known for such thin layers and the treatment conditions used. Therefore, four multilayers with different passivation times (1 min, 2.5 $\mathrm{min}, 4 \mathrm{~min}$, and $8 \mathrm{~min}$ ) were synthesized (Table 2). All other parameters were fixed, with the $\mathrm{N}_{2}$ flow being $7 \mathrm{sccm}$, the beam voltage $60 \mathrm{eV}$, and ion current density $0.68 \mathrm{~mA} / \mathrm{cm}^{2}$ (total ion current of $120 \mathrm{~mA}$ ).

\subsection{Soft X-ray reflectivity at $0.84 \mathrm{~nm}$}

Fig. 2 shows the reflectivity in the first Bragg peak at $0.84 \mathrm{~nm}$ of the multilayer systems with 50 periods versus different passivation times. The maximum reflectivity is observed for the reference multilayer

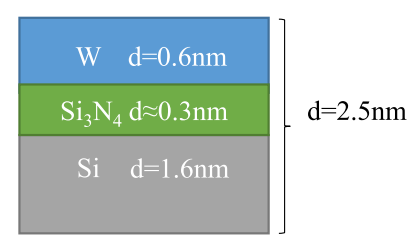

Fig. 1. Schematic representation of one period of the multilayer with a partially N-passivated Si layer. 
Table 2.

Structure IDs with the corresponding passivation times.

\begin{tabular}{llll}
\hline $\begin{array}{l}\text { Structure } \\
\text { ID }\end{array}$ & $\begin{array}{l}\text { Passivation time } \\
\text { (minutes) }\end{array}$ & $\begin{array}{l}\text { Number of periods } \\
\text { N }\end{array}$ & $\begin{array}{l}\text { Period value } \\
d(\mathrm{~nm})\end{array}$ \\
\hline T0 & 0 (reference) & 50,200 & 2.52 \\
T1 & 1 & 50 & 2.54 \\
T2 & 2.5 & 50 & 2.63 \\
T3 & 4 & 50,200 & 2.5 \\
T4 & 8 & 50 & 2.5 \\
\hline
\end{tabular}

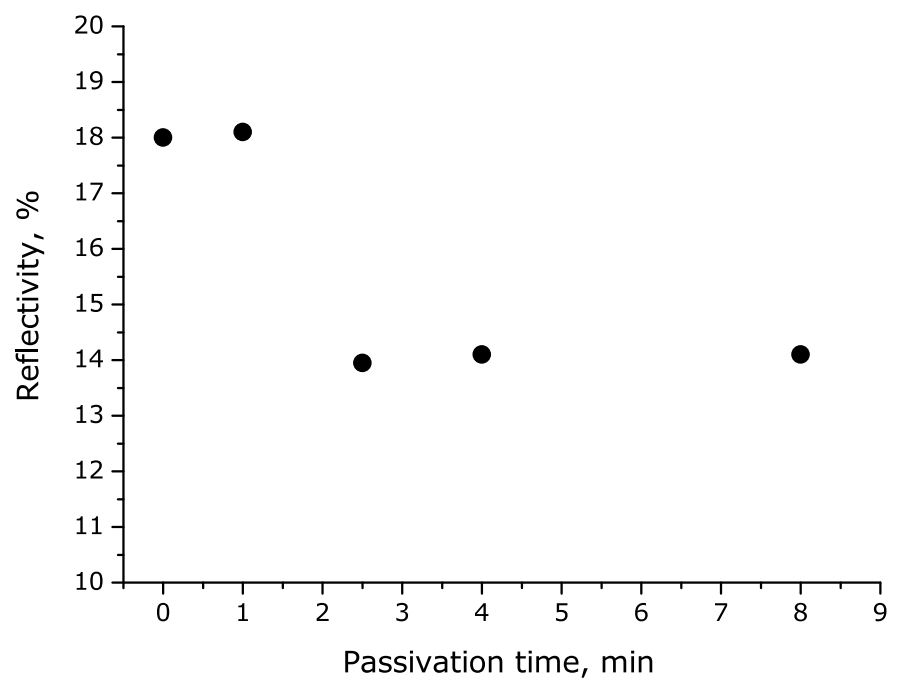

Fig. 2. Reflectivity of the passivated multilayer systems with various passivation times and the reference multilayer (time $=0$ ) at $0.84 \mathrm{~nm}$ and an angle of incidence $\theta \approx 9.7^{\circ}$. The period $d=2.5 \mathrm{~nm}$, the number of periods $N=50$ for all the multilayers.

(system T0) and the multilayer with one minute of passivation (system T1). They both have about the same reflectivity (of about 18\%) suggesting that no significant structural changes were present for 1-minute of passivation (structure T1). This observation is supported by the reflectivity measurements at $0.154 \mathrm{~nm}$ tat did not show any differences in the reflectivity curves either. However, for 2.5-minute passivation time (structure T2), a significant reduction in reflectivity at $0.84 \mathrm{~nm}$ was observed. The reflectivity remained the same for the longer passivation times of 4 and 8 min (structures T3 and T4). Note that this abrupt reduction in the reflectivity at $0.84 \mathrm{~nm}$ was accompanied by significant changes in the reflectivity at $0.154 \mathrm{~nm}$ as well, which will be discussed in Section 3.5.

A multilayer system with 4-minute passivation treatment and 200 periods was also fabricated. It had a reflectivity of about $29 \%$ at 0.84 $\mathrm{nm}$, which is $11 \%$ absolute value lower than the reflectivity of our W/Si reference multilayer system with 200 periods.

In the following sections, we investigate possible reasons for the reduction of the reflectivity at $0.84 \mathrm{~nm}$ in systems with passivated $\mathrm{Si}$.

\subsection{Diffuse scattering}

To determine the influence of passivation on roughness of the passivated multilayer systems, diffuse scattering at $\mathrm{CuK} \alpha$ radiation $(\lambda=$ $0.154 \mathrm{~nm}$ ) was measured. For that, we did rocking curve scans by fixing the detector at the center of a Bragg peak and rocking the sample in a wide angular range. A perfect structure will produce a very sharp peak in the specular direction only, while a structure with diffused scattering will show wide 'wings' around it. Rocking curve measurements from multilayer structures are described in more detail elsewhere [23]. It is common to measure diffuse scattering at higher Bragg orders to observe a wider range of angles. However, due to low intensity of the higher order peaks, all scans in this work were performed at a second Bragg peak at $3.2^{\circ}\left(2 \theta=6.4^{\circ}\right)$. The observed diffused scattering was similar for all the multilayers. For example, Fig. 3 shows a comparison of the $\mathrm{T} 1$ and T3 multilayer with the reference T0 multilayer.

The observed differences are not significant and indicate negligible differences in roughness of the measured samples. The passivation of Si did not affect the roughness to a sufficient extent to explain the drop in reflectivity at $0.84 \mathrm{~nm}$ for the passivated samples.

\subsection{X-ray photoelectron spectroscopy (XPS)}

In order to investigate an effect of the ion treatment on the composition and chemical state of the elements in the structures, the multilayers were analyzed by XPS. Fig. 4 shows an example of a sputter depth profile for the T3 structure (4 min of Si passivation). Since the probing depth of XPS is larger than the multilayer period, the sputter profile only shows a very weak modulation in the concentrations of $\mathrm{W}, \mathrm{Si}$ and $\mathrm{N}$. We used these profiles to determine an average concentration of $\mathrm{N}$ in every structure, which is plotted in Fig. 5. The concentration of $\mathrm{N}$ increases with the passivation time and reaches a saturation for 4- and 8-minute passivation. This implies that the passivation procedure was effective in nitridation the structure.

To determine the chemical state of the elements, the W4f and Si2p peaks were analyzed. In order to avoid intermixing induced formation of compounds by sputter depth profiling [24], the W4f and Si2p peaks were measured on the deposited samples without applying ion etching. Figs. 6 and 8 illustrate the measured W4f and Si2p spectra of the samples with selected passivation times compared to the reference sample without passivation. For the W4f peak, also a reference spectrum of a thick tungsten film is included, which was sputter cleaned by Ar ion-etching directly before the measurement.

In our previous work we showed that tungsten silicide formation results in a shift of the W4f peak to a lower binding energy by about 0.2 $\mathrm{eV}$ [18], compared to the reference position for bulk W. Since such small shifts are close to the resolution that can be obtained with laboratory XPS systems, we only provide a qualitative evaluation and do not attempt to deconvolute the W4f peak into different components. Fig. 7 shows that longer passivation leads to a higher fitted binding energy of the $\mathrm{W}_{4 f_{7 / 2}}$ peak, approaching the $\mathrm{W}$ reference binding energy. This implies some reduction of the tungsten silicide formation. However, since the shifts are close to the resolution of the laboratory XPS equipment, it is not possible to determine how strong the effect of silicide suppression is. Lastly, the sample deposited with 4-minute passivation shows some additional XPS intensity for the $\mathrm{W}_{4 f_{7 / 2}}$ peak around $32 \mathrm{eV}$,

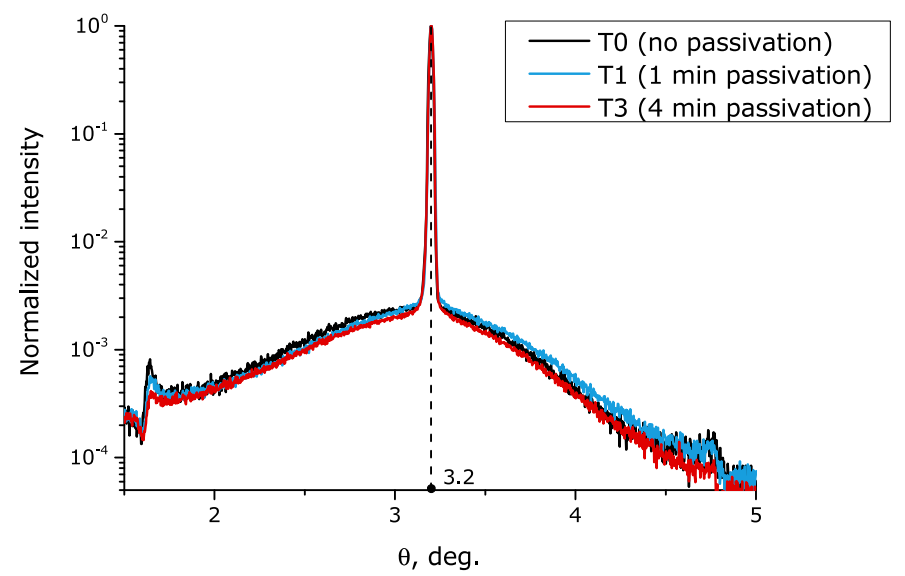

Fig. 3. Rocking curve scans for $\mathrm{W} / \mathrm{Si}(d=2.5 \mathrm{~nm}, N=50)$ multilayers deposited with and without passivation of the Si layers. Passivation times were 1 and $4 \mathrm{~min}$. The curves were normalized at the maximum intensity of the Bragg peak, for comparison. 


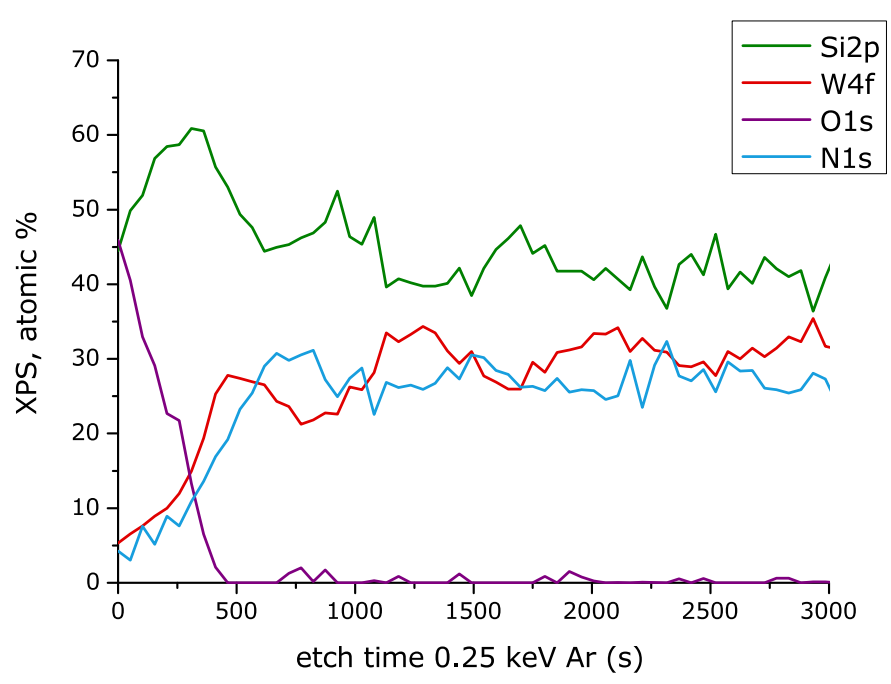

Fig. 4. XPS depth profiles of multilayer systems with 4 min of nitridation of Si layers.

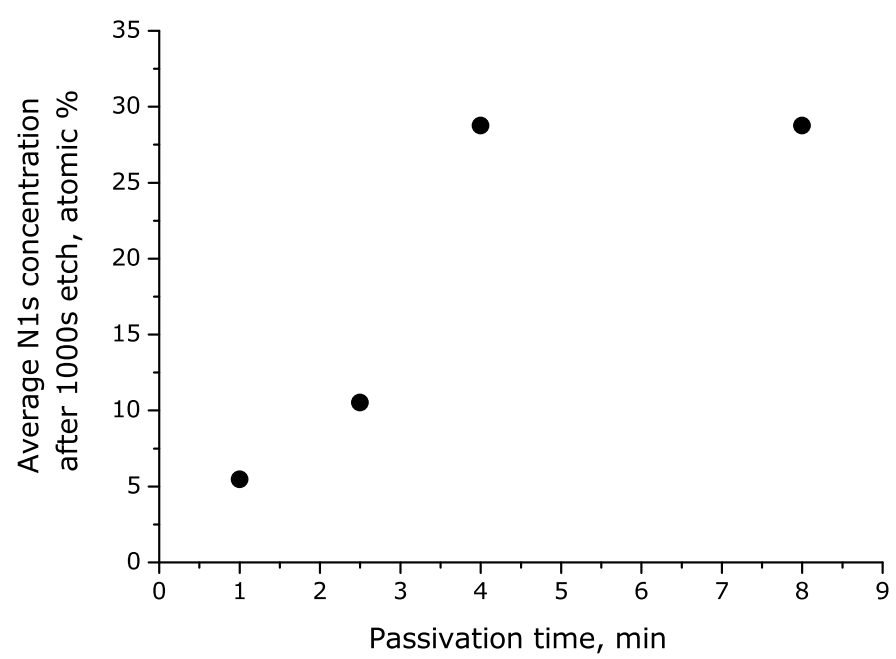

Fig. 5. Average concentration of nitrogen (N1S peak) in the samples with different time of passivation determined from the XPS depth profiles after a $1000 \mathrm{~s}$ etch time at $0.25 \mathrm{keV}$.

and for the $\mathrm{W}_{4} \mathrm{f}_{5 / 2}$ peak around $34 \mathrm{eV}$. The intensity around these binding energies is in line with $\mathrm{WN}_{\mathrm{x}}$ formation [25], which indicates that $\mathrm{W}$ may react with $\mathrm{N}$. This intensity strongly increases with the passivation time. This indicates that WNx is probably not a result of $\mathrm{W}$ reaction with $\mathrm{N}$ from the underlying SiNx layer. Instead nitrogen may penetrate through $\mathrm{Si}$ into the underlying $\mathrm{W}$ layer during passivation.

Fig. 8 illustrates Si2p spectral lines measured for selected samples. The W/Si reference mirror showed a slightly different background intensity due to differences in secondary electrons. Therefore, a Shirley background [26] was subtracted to facilitate comparison.

The peak around $99.5 \mathrm{eV}$ is assigned to elemental $\mathrm{Si}$ and $\mathrm{Si}$ in $\mathrm{WSi}_{\mathrm{x}}$ compounds. The peak around $103.5 \mathrm{eV}$ is due to native $\mathrm{SiO}_{2}$ formed because of the oxidation of the top surface after exposure to air. Due to the larger probing depth than the period of the multilayer system, it was possible to detect the formation of $\mathrm{SiN}_{\mathrm{x}}$ species, which contribute to the signal between 101.5 and $102 \mathrm{eV}$. However, it should be noted that substoichiometric $\mathrm{SiO}_{\mathrm{x}}$, present at the interface between $\mathrm{SiO}_{2}$ and $\mathrm{Si}$, may also result in intensity in the same binding energy range. It can be seen that, as the passivation time increases, the intensity in the 101.5 to $102 \mathrm{eV}$ binding energy range increases, confirming that $\mathrm{SiN}_{\mathrm{x}}$ is formed by the passivation treatment. A decrease of the $\mathrm{Si}$ peak around $99.5 \mathrm{eV}$

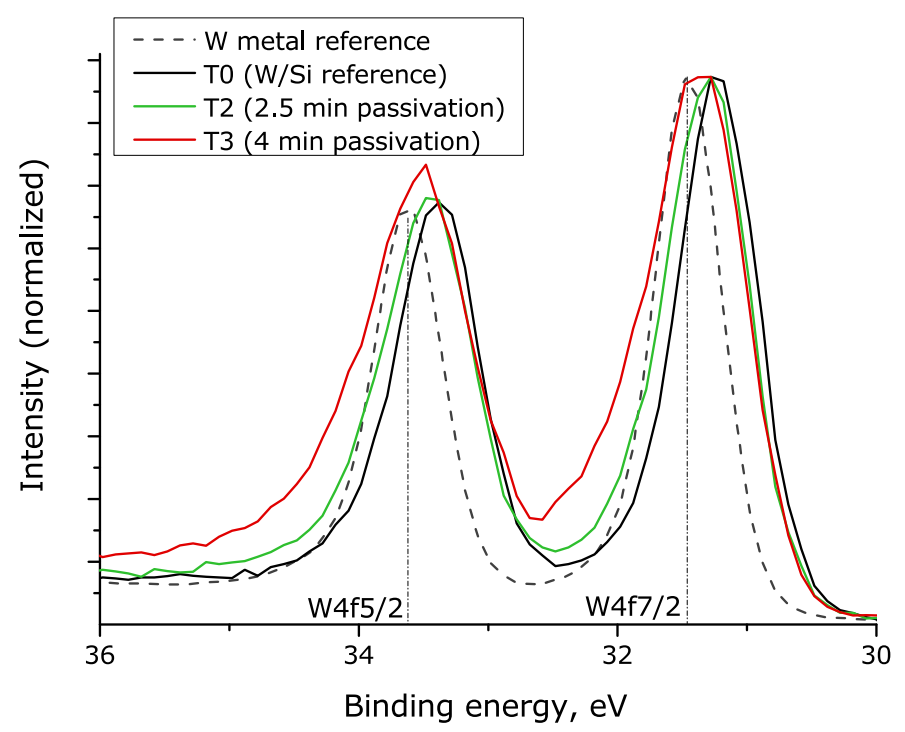

Fig. 6. The measured XPS W4f doublet for a multilayer with 2.5-minute (red line) and 4-minute (green line) passivation compared to a multilayer without passivation (blue) and a $\mathrm{W}$ reference (dashed line). The signals are normalized to the $\mathrm{W}_{4} \mathrm{f}_{7 / 2}$ peak. (For interpretation of the references to color in this figure legend, the reader is referred to the web version of this article.)

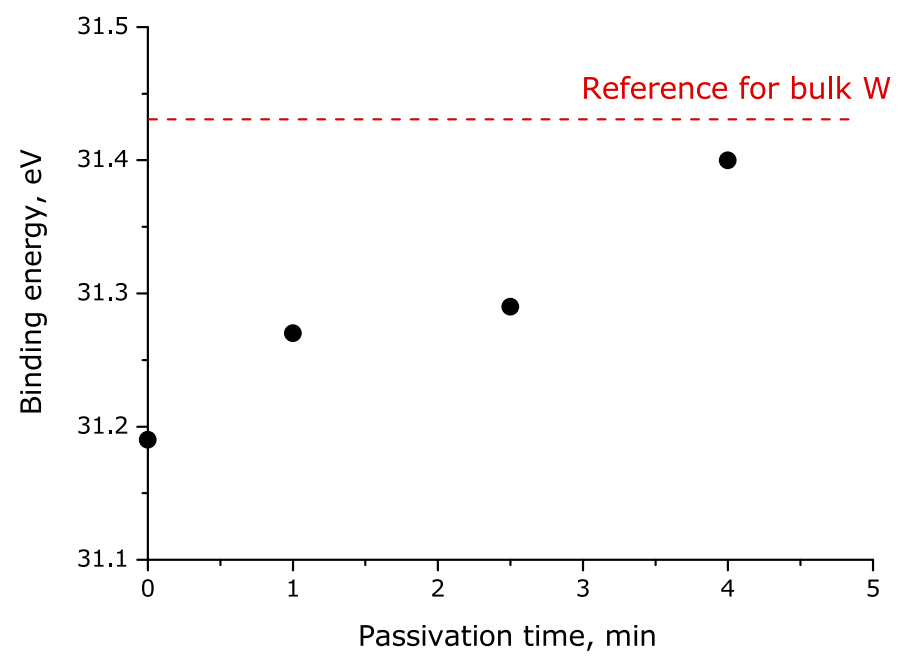

Fig. 7. The fitted peak position of the XPS $W 4 f_{7 / 2}$ peak as function of passivation time.

can be explained by the process of conversion of $\mathrm{Si}$ to $\mathrm{SiN}_{\mathrm{x}}$.

Note that possible $\mathrm{Ar}^{+}$implantation in Si layers during passivation treatment could also affect the reflectivity of the multilayer system [27]. However, XPS analysis did not reveal any presence of $\mathrm{Ar}^{+}$in our samples.

\subsection{Grazing incidence X-ray reflectivity and X-ray fluorescence analysis}

Although XPS analysis gave information about the presence of nitrogen and chemical interactions taking place in the systems upon the passivation treatment, the limited depth resolution of XPS analysis did not give accurate information about the in-depth distribution of the materials. To analyze this, we used X-ray reflectivity measurements (GIXR) in combination with X-ray fluorescence modulated by the X-ray standing wave analysis (F-XSW). This combination allowed us to reduce the cross-correlations between the model parameters during the analysis of X-ray reflectivity by adding the X-ray fluorescence dataset that is 


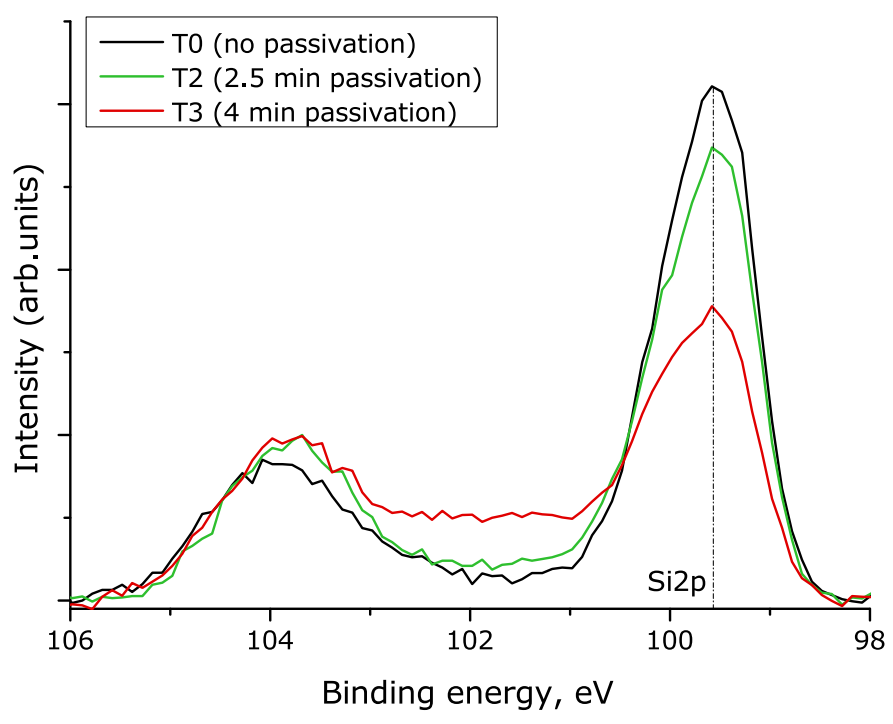

Fig. 8. Si $2 p$ peaks for the multilayer structure with 2.5-minute passivation (red), 4-minute passivation (green), and a reference structure without passivation (blue). A Shirley background was subtracted from all spectra. No normalization of intensities was applied. (For interpretation of the references to color in this figure legend, the reader is referred to the web version of this article.)

sensitive to the phase of the reflected waves. This approach partially solves [28] the well-known phase problem of X-ray reflectivity [29,30]. We could not measure the fluorescence signal from $\mathrm{N}$ in our laboratory equipment. The fluorescence signal from $\mathrm{Si}$ from the multilayer would be mixed with the signal from the Si substrate. So only the fluorescence signal from $\mathrm{W}$ was measured. To fit the data, a so called free-form analysis was used [22]. Unlike the conventional model-based fitting method, it allows for more freedom in modelling interface profiles, which is essential for short-period multilayers where interaction zones are substantial compared to the period of the structure. We analyzed the structures T0 (no passivation), T2 (2.5-minute passivation), and T3 (4-minute passivation). The data analysis was performed as discussed in [21] with implementation of free-form multilayer parametrization approach described in [31]. The period of the reference multilayer T0 was modeled as a set of sublayers where the concentration of the elements in the sublayers, and consequently the optical constants were allowed to vary between $\mathrm{W}$ and $\mathrm{Si}$. To account for nitridation for structures T2 (2.5-minute passivation) and T3 (4-minute passivation) the element concentration and layer density was allowed to vary from $\mathrm{W}$ to SiN to pure Si. Examples of the achieved fits are shown in Fig. 9 (for

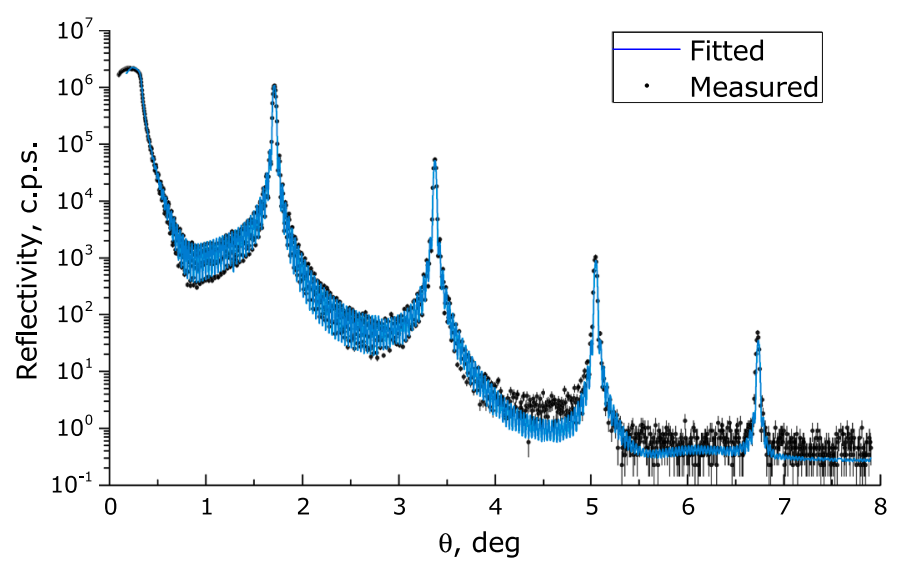

Fig. 9. Measured (dots) and simulated X-ray reflectivity data based on best-fit solutions (lines) for the T2 (2.5-minute passivation) multilayer.
GIXR) and Fig. 10 (for F-XSW). The fitting curves are in a good agreement with the measured data for all three multilayer systems.

As a result of this analysis, we obtained a set of the concentrations of the elements, and densities per sub layer. These were used for the calculation of the optical constants at $0.84 \mathrm{~nm}$ wavelength as described in [32]. Fig. 11 shows the real part of the optical constant profiles obtained for T0, T2, and T3, as derived from the simultaneous fit described above. These profiles were used to calculate the at-wavelength reflectivity shown in Fig. 12. A good match between the measured and calculated reflectivity was obtained.

Note that a small change in the period of the structure T3 is observed. This may be ascribed to the fact that the $0.154 \mathrm{~nm} \mathrm{X}$-ray reflectivity measurements were done about two years after the $0.84 \mathrm{~nm}$ wavelength $\mathrm{X}$-ray reflectivity measurements. We suggest that interdiffusion process that may continue in the multilayer after deposition could have been more significant in the T3 structure. This phenomenon calls for a separate research.

\section{Discussion}

The passivation treatment procedure of using reactive nitrogen clearly resulted in some reduction of the silicide formation, as proven by XPS. However, it did not cause a reflectivity improvement at $0.84 \mathrm{~nm}$ wavelength. It is remarkable that the 1 -minute passivation did not result in any changes in reflectivity detectable by either $0.154 \mathrm{~nm}$ or $0.84 \mathrm{~nm}$ measurements. This is in spite of the fact that the XPS measurements did detect some amount of $\mathrm{N}$ present in the systems. It could be argued that this treatment did not produce a continuous passivated Si layer, with the chemical processes at the $\mathrm{W}$-on-Si interface being not affected significantly. However, the 2.5-minute passivation treatment did result in a pronounced reduction in the reflectivity at $0.84 \mathrm{~nm}$. This is in spite of the fact that it leads to the formation of sharper interfaces, as seen in the reconstructed refractive index profile (Fig. 11). Still, this also showed a reduced contrast between the reflector and spacer layer because of an elevated plateau formed in the refractive index observed in the spacer layer. The occurrence of this plateau can be ascribed to the formation of a nitrided Si layer $\left(\mathrm{Si}_{\mathrm{x}} \mathrm{N}_{\mathrm{y}}\right)$. Evidently, the observed improvement in the interface profile is compensated by the reduced optical contrast due to the formation of a thick $\mathrm{Si}_{\mathrm{x}} \mathrm{N}_{\mathrm{y}}$ layer, resulting in a reduction of the

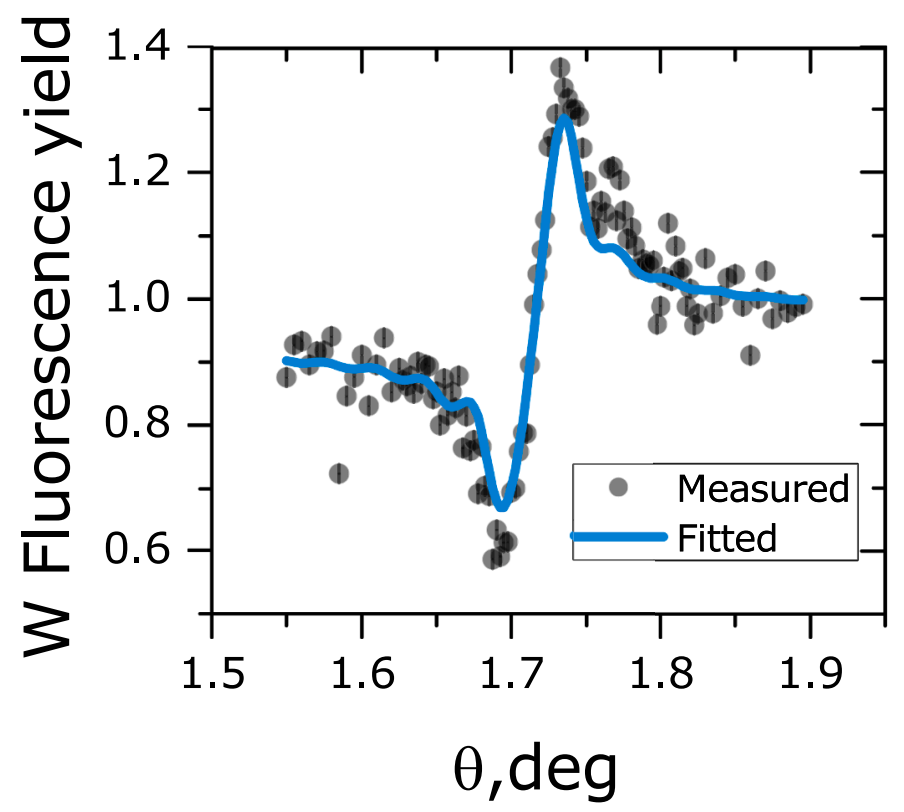

Fig. 10. Measured (dots) and simulated (lines) angular dependent X-ray fluorescence yields around the first Bragg peak $\left(\theta=1.71^{\circ}\right)$ for $\mathrm{W}$ for the T2 (2.5minute passivation) multilayer sample. 


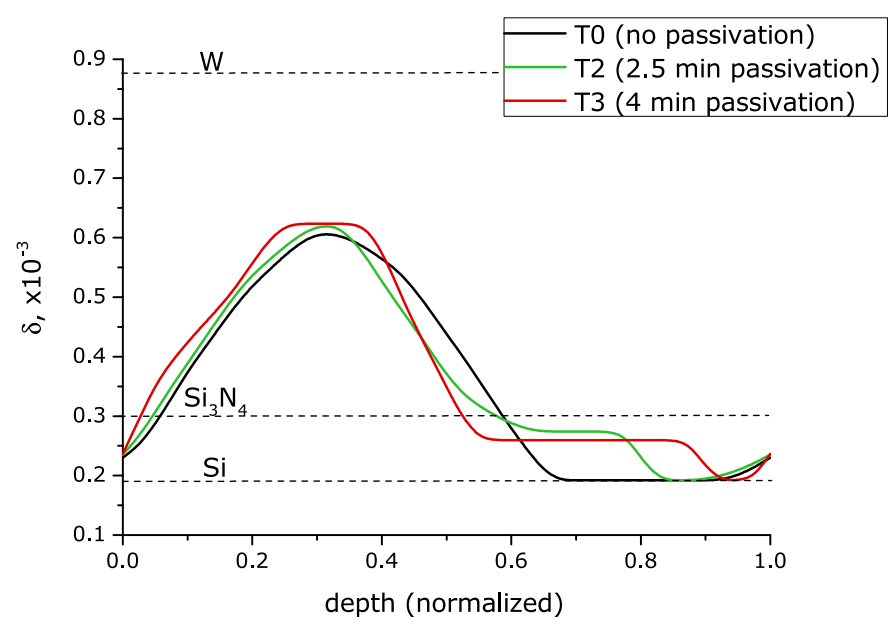

Fig. 11. The real part of the optical constant decrements obtained for $0.84 \mathrm{~nm}$ wavelength for the structures T0 (no passivation), T2 (2.5-minute passivation), and T3 (4-minute passivation). Due to slightly deviating periods of the samples within about $0.1 \mathrm{~nm}$ (see Table 2), the depth values were normalized to corresponding period values of each sample.

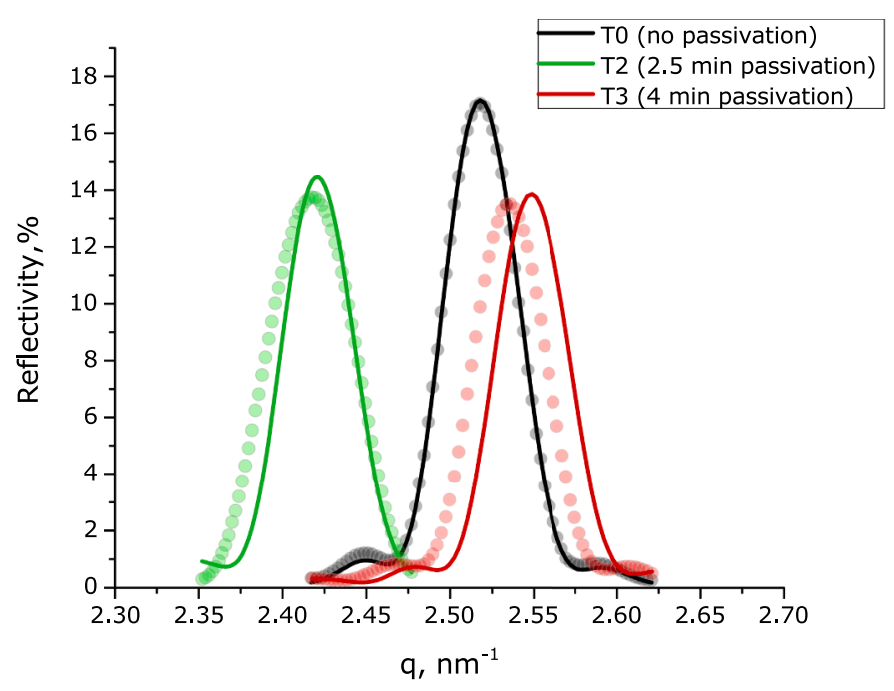

Fig. 12. Measured and simulated reflectivity at $0.84 \mathrm{~nm}$ using the optical constant profiles shown in Fig. 11 for the T0, T2 and T3 multilayer systems.

reflectivity at $0.84 \mathrm{~nm}$. The longer passivation time (4-minutes) improves the interfaces further and defines the reflecting layer better. The latter is visible by a plateau formed in the reflecting layer. At the same time, the thickness of the formed $\mathrm{SiN}_{\mathrm{X}}$ layer increases as well. Furthermore, the formation of $\mathrm{WN}_{\mathrm{x}}$ species is observed by XPS for the 4-minute passivation treatment. This could be the reason the optical density of the absorber does not increase in spite of the formation of sharper interfaces.

Summarizing, it can be concluded that passivation of Si by nitrogen can result in improvement of the interfaces in W/Si multilayers. However, a gentler passivation procedure could be explored to avoid excessive penetration of nitrogen in the Si-layer which may reduce the thickness of the $\mathrm{Si}_{\mathrm{x}} \mathrm{N}_{\mathrm{y}}$ layer.

\section{Conclusion}

We explored partial nitridation of $\mathrm{W} / \mathrm{Si}$ multilayer systems with a $2.5 \mathrm{~nm}$ layer periodicity. The purpose was to reduce the optically unfavorable tungsten silicide formation in W/Si systems leading to X-ray reflectivity loss. The multilayers were deposited by magnetron sputtering, and nitridation was applied directly after the deposition of every Si layer by exposing the Si surfaces to reactive nitrogen species from a Kaufman ion source operated at $60 \mathrm{~V}$. The passivation was in the range of 1 to $8 \mathrm{~min}$.

The passivation treatment of 1-minute did not result in any changes in X-ray reflectivity, as detectable at 0.154 or $0.84 \mathrm{~nm}$ wavelength. The treatment of 2.5-minute and longer did result in a reduced tungsten silicide formation (observed by XPS) and improved sharpness of the interfaces (detected by $0.154 \mathrm{~nm}$ X-ray reflectometry). However, this was accompanied by a pronounced reduction in the reflectivity at 0.84 $\mathrm{nm}$. The refractive index profile, reconstructed from $0.154 \mathrm{~nm}$ X-ray reflectivity, revealed the formation of an elevated plateau in the refractive index of the spacer layer. This was attributed to the formation of a thicker than $0.5 \mathrm{~nm}$ silicon nitride layer that reduced the optical contrast of the multilayer at $0.84 \mathrm{~nm}$.

We conclude that passivation of $\mathrm{Si}$ using nitrogen can result in improvement of interfaces in W/Si multilayers. However, in the tested conditions, this improvement was counterbalanced by a reduced optical contrast due to a relatively thick silicon nitride layer. This suggests that gentler passivation procedures should be tried to reduce nitrogen penetration in Si.

\section{CRediT authorship contribution statement}

R.V. Medvedev: Conceptualization, Methodology, Software, Writing - original draft, Investigation, Writing - review \& editing. C.P. Hendrikx: Software, Formal analysis. J.M. Sturm: Investigation, Writing - review \& editing. S.N. Yakunin: Resources. I.A. Makhotkin: Investigation, Software, Formal analysis. A.E. Yakshin: Supervision, Methodology, Writing - review \& editing. F. Bijkerk: Supervision, Project administration.

\section{Declaration of Competing Interest}

The authors declare that they have no known competing financial interests or personal relationships that could have appeared to influence the work reported in this paper.

\section{Acknowledgements}

This work has been carried out in the frame of the Industrial Partnership Program 'X-tools', project number 741.018.301, funded by the Netherlands Organization for Scientific Research, ASML, Carl Zeiss SMT, and Malvern Panalytical. We acknowledge the support of the Industrial Focus Group XUV Optics at the MESA+ Institute for Nanotechnology at the University of Twente. The Physikalisch-Technische Bundesanstalt (PTB) EUV-radiometry group is acknowledged for doing the reflectivity measurements. S.N. Yakunin acknowledges the funding of Russian Foundation for Basic Research (RFBR), project number 19-29-12038.

\section{References}

[1] R.V Medvedev, K.V Nikolaev, A.A. Zameshin, D. Ijpes, I.A. Makhotkin, S. N. Yakunin, A.E. Yakshin, F. Bijkerk, Low-energy ion polishing of Si in W/Si soft Xray multilayer structures, J. Appl. Phys. 126 (2019), 045302, https://doi.org/ 10.1063/1.5097378.

[2] C. Liu, R. Conley, A.T. Macrander, T.J. Graber, C. Morawe, C. Borel, E.M. Dufresne, Small-d-spacing $\mathrm{WSi}_{2}$ /Si narrow bandpass multilayers, X-ray Sources Opt. 5537 (2004) 154, https://doi.org/10.1117/12.561399.

[3] A.A. Zameshin, R.V Medvedev, A.E. Yakshin, F. Bijkerk, Interface formation in W /Si multilayers, Thin Solid Films (2021), https://doi.org/10.1016/j. tsf.2021.138569.

[4] T.P. Ma, Making silicon nitride film a viable gate dielectric, IEEE Trans. Electron. Devices 45 (1998) 680-690, https://doi.org/10.1109/16.661229.

[5] S. Guruvenket, J. Ghatak, P.V. Satyam, G.M. Rao, Characterization of bias magnetron-sputtered silicon nitride films, Thin Solid Films 478 (2005) 256-260, https://doi.org/10.1016/j.tsf.2004.10.031.

[6] B.C. Kim, H. Kang, C.Y. Kim, J.W. Chung, Ion beam nitridation of a Si(111) surface: effects of ion reactivity and thermal treatment, Surf. Sci. 301 (1994) 295-305, https://doi.org/10.1016/0039-6028(94)91309-9. 
[7] I.A. Makhotkin, E. Zoethout, R. van de Kruijs, S.N. Yakunin, E. Louis, A. M. Yakunin, V. Banine, S. Müllender, F. Bijkerk, Short period La/B and LaN/B multilayer mirrors for $\sim 68 \mathrm{~nm}$ wavelength, Opt. Express 21 (2013) 29894, https:// doi.org/10.1364/oe.21.029894.

[8] D.S. Kuznetsov, A.E. Yakshin, J.M. Sturm, R.W.E. van de Kruijs, E. Louis, F. Bijkerk, High-reflectance La/B-based multilayer mirror for $6 \mathrm{x} \mathrm{nm}$ wavelength, Opt. Lett. 40 (2015) 3778, https://doi.org/10.1364/OL.40.003778.

[9] D.S. Kuznetsov, A.E. Yakshin, J.M. Sturm, R.W.E. Van De Kruijs, F. Bijkerk, Structure of high-reflectance La/B-based multilayer mirrors with partial la nitridation, AIP Adv. 6 (2016), https://doi.org/10.1063/1.4968175.

[10] T. Tsarfati, R.W.E. Van De Kruijs, E. Zoethout, E. Louis, F. Bijkerk, Nitridation and contrast of B4C/La interfaces and X-ray multilayer optics, Thin Solid Films 518 (2010) 7249-7252, https://doi.org/10.1016/j.tsf.2010.04.088.

[11] T. Tsarfati, E. Zoethout, E. Louis, R. van de Kruijs, A. Yakshin, S. Müllender, F. Bijkerk, Improved contrast and reflectivity of multilayer reflective optics for wavelengths beyond the extreme UV, Altern. Lithogr. Technol. 7271 (2009) 72713V, https://doi.org/10.1117/12.824434.

[12] Q. Huang, Y. Liu, Y. Yang, R. Qi, Y. Feng, I.V. Kozhevnikov, W. Li, Z. Zhang, H. Jiang, L. Zhang, A. Li, J. Wang, Z. Wang, Nitridated Ru/B4C multilayer mirrors with improved interface structure, zero stress, and enhanced hard X-ray reflectance, Opt. Express 26 (2018) 21803, https://doi.org/10.1364/ oe.26.021803.

[13] Y. Wang, Q. Huang, Q. Yi, I.V. Kozhevnikov, R. Qi, M. Wen, P. Jonnard, J. Zhang, A. Giglia, Z. Zhang, Z. Wang, Nitridated $\mathrm{Pd} / \mathrm{B}_{4} \mathrm{C}$ multilayer mirrors for soft X-ray region: internal structure and aging effects, Opt. Express 25 (2017) 7749, https:// doi.org/10.1364/oe.25.007749.

[14] M. Wen, Q. Huang, S. Ma, W. Li, R. She, J. Peng, A. Giglia, I.V. Kozhevnikov, H. Feng, Z. Zhang, Z. Wang, Improvement of interface structure and polarization performance of $\mathrm{Co} / \mathrm{C}$ multilayers by incorporation of nitrogen, Opt. Express 24 (2016) 27166, https://doi.org/10.1364/oe.24.027166.

[15] K.H. Park, B.C. Kim, H. Kang, Silicon nitride formation by low energy $\mathrm{N}^{+}$and $\mathrm{H} 2+$ ion beams, J. Chem. Phys. 97 (1992) 2742-2749, https://doi.org/10.1063/ 1.463064 .

[16] I. Kusunoki, T. Takaoka, Y. Igari, K. Ohtsuka, Nitridation of a Si(100) surface by 100-1000 eV N+2 ion beams, J. Chem. Phys. 8238 (1994) 101, https://doi.org/ 10.1063/1.468194.

[17] O.C. Hellman, N. Herbots, O. Vancauwenberghe, Kinetics of ion beam nitridation (IBN) of Si and of MBE-grown Ge and SixGe1-x alloys: the role of ion energy, ion dose and substrate temperature, Nucl. Inst. Methods Phys. Res. B. 67 (1992) 301-307, https://doi.org/10.1016/0168-583X(92)95821-8.

[18] R.V. Medvedev, A.A. Zameshin, J.M. Sturm, A.E. Yakshin, F. Bijkerk, W/B short period multilayer structures for soft X-rays, AIP Adv. 10 (2020), https://doi.org/ 10.1063/1.5143397.

[19] D.E. Savage, Y.H. Phang, J.J. Rownd, J.F. MacKay, M.G. Lagally, Determination of interfacial roughness correlation in W/C multilayer films: comparison using soft and hard X-ray diffraction, J. Appl. Phys. 74 (1993) 6158-6164, https://doi.org/ $10.1063 / 1.355182$.
[20] P.J. Cumpson, M.P. Seah, Elastic scattering corrections in AES and XPS. II. Estimating attenuation lengths and conditions required for their valid use in overlayer/substrate experiments, Surf. Interface Anal. 25 (1997) 430-446, https:// doi.org/10.1002/(SICI)1096-9918(199706)25:6<430::AID-SIA254>3.0.CO;2-7.

[21] S.N. Yakunin, I.A. Makhotkin, R.W.E. Van De Kruijs, M.A. Chuev, E.M. Pashaev, E. Zoethout, E. Louis, S.Y. Seregin, I.A. Subbotin, D.V. Novikov, F. Bijkerk, M. V. Kovalchuk, Model independent X-ray standing wave analysis of periodic multilayer structures, J. Appl. Phys. (2014) 115, https://doi.org/10.1063/ 1.4869540 .

[22] A. Zameshin, I.A. Makhotkin, S.N. Yakunin, R.W.E. Van De Kruijs, A.E. Yakshin, F. Bijkerk, Reconstruction of interfaces of periodic multilayers from X-ray reflectivity using a free-form approach, J. Appl. Crystallogr. 49 (2016) 1300-1307, https://doi.org/10.1107/S160057671601044X.

[23] D.E. Savage, J. Kleiner, N. Schimke, Y.H. Phang, T. Jankowski, J. Jacobs, R. Kariotis, M.G. Lagally, Determination of roughness correlations in multilayer films for X-ray mirrors, J. Appl. Phys. 69 (1991) 1411-1424, https://doi.org/ 10.1063/1.347281.

[24] B.R. Chakraborty, S. Hofmann, Ion bombardment induced silicide formation during sputter depth profiling of $\mathrm{Ta} / \mathrm{Si}$ multilayer thin film structure as studied by $\mathrm{X}$-ray photoelectron spectroscopy and auger electron spectroscopy, Thin Solid Films 204 (1991) 163-174, https://doi.org/10.1016/0040-6090(91)90502-O.

[25] R.J. Colton, J.W. Rabalais, Electronic structure to tungsten and some of its borides, carbides, nitrides, and oxides by X-ray electron spectroscopy, Inorg. Chem. 15 (1976) 236-238, https://doi.org/10.1021/ic50155a049.

[26] D.A. Shirley, High-resolution X-ray photoemission spectrum of the valence bands of gold, Phys. Rev. B. 5 (1972) 4709-4714, https://doi.org/10.1103/ PhysRevB.5.4709.

[27] E. Spiller, S.L. Baker, P.B. Mirkarimi, V. Sperry, E.M. Gullikson, D.G. Stearns, Highperformance Mo-Si multilayer coatings for extreme-ultraviolet lithography by ionbeam deposition, Appl. Opt. 42 (2003) 4049-4058, https://doi.org/10.1364/ ao.42.004049.

[28] M.J. Bedzyk, G.M. Bommarito, J.S. Schildkraut, X-ray standing waves at a reflecting mirror surface, Phys. Rev. Lett. 62 (1989) 1376-1379, https://doi.org/ 10.1103/PhysRevLett.62.1376.

[29] K.M. Zimmermann, M. Tolan, R. Weber, J. Stettner, A.K. Doerr, W. Press, Phase determination of X-ray reflection coefficients, Phys. Rev. B 62 (2000) 10377-10382, https://doi.org/10.1103/PhysRevB.62.10377.

[30] J. Ehlers, K. Hepp, R. Beig, W. Domcke, U. Frisch, W. Hillebrandt, R.L. Jaffe, X-ray and Neutron Reflectivity. Principles and Applications, Springer, 2009, https://doi. org/10.1007/978-3-540-88588-7.

[31] S.Y. I.A. Makhotkin, Model Independent Grazing Incidence X-ray Reflectivity, US Pat. US10416101B2. 2 (2016).

[32] S.N. Yakunin, I.A. Makhotkin, K.V. Nikolaev, R.W.E. van de Kruijs, M.A. Chuev, F. Bijkerk, Combined EUV reflectance and X-ray reflectivity data analysis of periodic multilayer structures, Opt. Express 22 (2014) 20076, https://doi.org/ 10.1364/oe.22.020076. 